\title{
Incorporación de nitrógeno y fósforo por Sarcocornia perennis (Miller) A. J. Scott en concentraciones reales en el estuario del río Palmones
}

\author{
Rocío Muñoz $^{1, *}$ and F. Xavier Niell ${ }^{1}$ \\ ${ }^{1}$ Departamento de Ecología, Facultad de Ciencias, Universidad de Málaga, Campus de teatinos s/n, 29071 Mála- \\ ga, España.
}

* Autor responsable de la correspondencia: rociomj@uma.es

Recibido: 26/11/08

Aceptado: 4/5/09

\begin{abstract}
Nitrogen and phosphorus uptake by Sarcocornia perennis (Miller) A. J. Scott, at natural concentrations in the Palmones river estuary

Sarcocornia perennis (Miller) A. J. Scott is a Chenopodiaceae dominant in salt marshes of Palmones river estuary (Spain). It forms dense and extended meadows which act as an active nutrient sink between land and sea. The capacity of nutrient uptake has been studied at natural concentrations found in field surveys. $V_{\max }, K_{m}$, and compensation point $\left(C_{\min }\right)$ for nutrients have been obtained for $\mathrm{NO}_{3}^{-}, \mathrm{PO}_{4}^{-3}$, and $\mathrm{NH}_{4}^{+}$.

Nitrate is limitant for the plant, but the nitrogen deficiency is replaced by $\mathrm{NH}_{4}^{+}$as a nitrogen source. The advantage of Sarcocornia over other macrophytes such as Spartina (Spartina alterniflora and Spartina patens) has been discussed.
\end{abstract}

Key words: Sarcocornia perennis, nutrient, assimilation kinetics.

\section{RESUMEN}

Incorporación de nitrógeno y fósforo por Sarcocornia perennis (Miller) A. J. Scott, en concentraciones reales en el estuario del río Palmones

Sarcocornia perennis (Miller) A. J. Scott es una Quenopodiácea dominante en las marismas saladas de Estuario del Río Palmones (España). Esta especie forma extensas y densas praderas las cuales actúan como sumidero de nutrientes entre la tierra y el mar. Se ha estudiado la capacidad de incorporar nutrientes en concentraciones reales encontradas en el medio por esta planta. Se ha obtenido la $\mathrm{V}_{\text {max }}, \mathrm{K}_{\mathrm{m}}, y \mathrm{C}_{\text {min }}$ para cada nutriente $\left(\mathrm{NO}_{3}^{-}, \mathrm{PO}_{4}^{-3}\right.$ y $\left.\mathrm{NH}_{4}^{+}\right)$.

El $\mathrm{NO}_{3}^{-}$se encuentra en concentraciones limitantes para la planta, pero esta deficiencia de nitrógeno es solventada por el $\mathrm{NH}_{4}^{+}$como fuente de nitrógeno. Las ventajas de Sarcocornia sobre otros macrófitos como Spartina (Spartina alterniflora and Spartina patens) son discutidas.

Palabras clave: Sarcocornia perennis, nutriente, cinética de asimilacion.

\section{INTRODUCCIÓN}

El estuario del río Palmones se ubica en la Bahía de Algeciras (Fig. 1). Desde hace años, este estuario ha estado sometido a una progresiva eutrofización (Niell et al., 1996, Clavero et al., 1999, Avilés,
2002; Palomo, 2004) debido al incremento en la entrada de nutrientes, principalmente nitrato $\mathrm{y}$ fosfatos producidos por la actividad humana.

El nitrógeno es el nutriente mineral que las plantas necesitan en mayores cantidades y uno de los factores frecuentemente limitantes para el cre- 


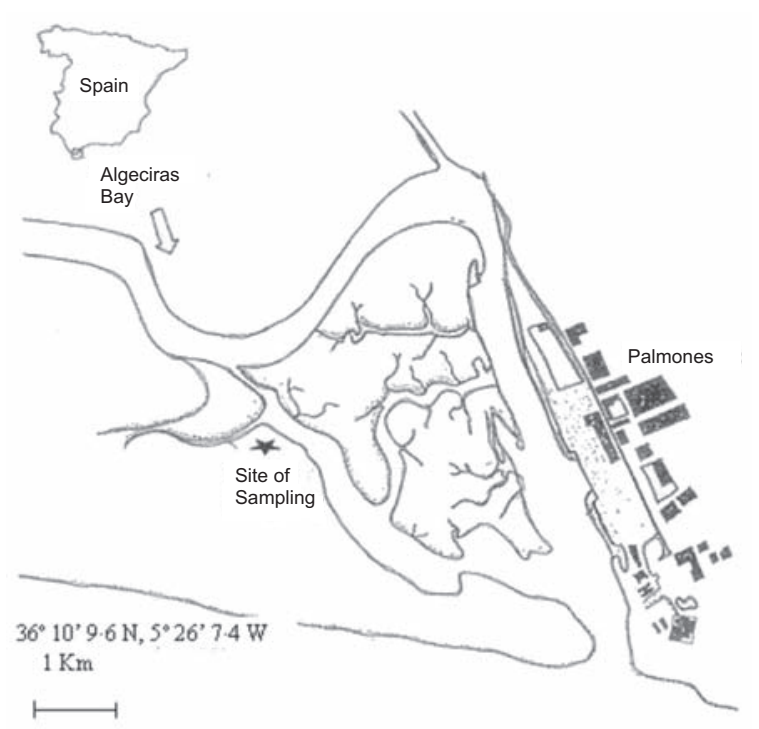

Figura 1. Estuario del río Palmones y zona de muestreo. Palmones River Estuary and sampling zone.

cimiento (Mendelson, 1979; Debusk y Reddy, 1987; Caetano et al., 2007). La mayoría de las plantas toman el nitrógeno a través de las raíces desde el suelo como $\mathrm{NO}_{3}^{-}$ó $\mathrm{NH}_{4}^{+}$, mostrando una alta afinidad por el $\mathrm{NH}_{4}^{+}$como forma de nitrógeno para ser asimilado (Morris, 1980; Morris, 1984; Jampeetong y Brix., 2009 (en prensa); Simas y Ferreira, 2007) mientras que el $\mathrm{NO}_{3}^{-}$juega un papel menor en la nutrición de la planta, aunque cuando está disponible, las plantas muestran la capacidad de asimilarlo (Mendelsson, 1979). La preferencia por el amonio podría ser debida a que la energía requerida para la reducción de nitrato se ahorra mediante la incorporación de amonio (Rosenberg y Ramus, 1984), aunque según diversos estudios, la capacidad de almacenamiento del amonio en la planta es limitado debido a su efecto tóxico (Waite y Mitchell, 1972; Haines y Wheeler, 1978; Lotze y Schramm, 2000; Cohem et al., 2004).

Por otro lado, la incorporación de $\mathrm{PO}_{4}^{3-}$ por las plantas, depende en gran parte del contenido interno en fosfato que presente la planta (Imaoka y Teranishi, 1988), y además, algunos estudios han mostrado como por ejemplo en plantas de soja, la presencia de amonio en altas concentraciones $(>500 \mu \mathrm{M})$ puede interferir en la incorporación del fosfato inhibiéndolo (Rayar y Hai, 1977), o como en el caso del maíz, incrementando su incorporación (Smith y Jackson, 1998).

En los estuarios los aportes de nutrientes llegan desde el río hacia el sedimento, donde son retenidos, e incorporados por la vegetación. Los procesos de intercambio que tienen lugar entre el sedimento y el agua que inunda la marisma influyen de manera determinante sobre los nutrientes que se encuentran en el agua intersticial. Así el fosfato en el agua intersticial de la marisma varía en un rango de concentraciones de $1.2 \pm 0.2 \mu \mathrm{M}$ y $112.7 \pm 45.7 \mu \mathrm{M}$ según Clavero et al. (2000); Izquierdo (2001); Avilés et al. (2002) y Palomo (2004).

Respecto de las formas de nitrógeno disponibles, en la marisma del río Palmones el agua intersticial del sedimento se encuentra bastante enriquecida en las formas solubles de $\mathrm{N}$ inorgánico, principalmente en amonio. Las concentraciones de nitrato encontradas en el agua intersticial del estuario oscilan entre los $0.04 \pm 0.01 \mu \mathrm{M}$ y $17.45 \pm 3.87 \mu \mathrm{M}$. Sin embargo, el amonio que es siempre la forma inorgánica dominante en marismas (De Laune et al., 1983), llega a encontrarse en concentraciones que van desde los $134 \pm 82 \mu \mathrm{M}$ hasta los $1505 \pm 483 \mu \mathrm{M}$ (Palomo, 2004).

Otros factores que deben ser considerados en el estudio de la incorporación de nutrientes además de la concentración de nutrientes en el suelo son las condiciones edáficas, ya que éstas pueden modificar las cinéticas de incorporación de nutrientes. Estas condiciones edáficas comprenden principalmente el estado de aireación del suelo (aerobia/anoxia) y la salinidad.

Está bien establecido que las tasas de incorporación de nutrientes son dependientes de un buen suministro de oxígeno a las raíces (Vlamis y Davis, 1944; Hammond et al., 1955; Hopkins, 1956). Así, en los suelos inundados o con poca aireación, se ha demostrado como las plantas tienen la capacidad de suministrar oxígeno internamente al sistema radicular (Rao y Mikkelsen, 1977). En Palmones, debido a los largos periodos de encharcamiento a los que está sometido el sedimento durante el invierno, el oxígeno se agota a $1 \mathrm{~cm}$ de profundidad, mientras que en verano el oxígeno que se encuentra en el sedimento es mucho mayor $(100 \mu$ moles/l) aunque se reduce a la mitad a partir de $l o s 2 \mathrm{~cm}$ de profundidad (Palomo, 2004). 
La salinidad es otra variable que puede modificar las tasas de incorporación de los nutrientes ya que el incremento de la salinidad hace decrecer las tasas de incorporación de los nutrientes (Fied et al., 1965; Helal et al., 1975) posiblemente por la competición entre iones de cargas similares. La salinidad en los suelos de la marisma del Palmones oscila intensamente durante todo el año como consecuencia de la frecuencia de inundación por la marea y de las tasas de evaporación (Palomo, 2004). Así se encuentran rangos de salinidad que van desde 30 hasta los 90 .

El principal objetivo de este trabajo es estudiar la incorporación de nutrientes en concentraciones reales que se dan en la marisma del estuario del río Palmones por la quenopodiácea $S$. perennis; para así discutir el papel real de esta especie en la dinámica de los nutrientes en este sistema estuárico.

\section{MATERIAL Y MÉTODOS}

Sarcocornia perennis se recolectó en las marismas del estuario del río Palmones. Las plantas fueron mantenidas en cultivos hidropónicos con solución Hoagland modificada (Epstein, 1972), a temperatura constante de $25^{\circ} \mathrm{C}$, con un flujo fotónico de $180 \mu \mathrm{mol} \mathrm{m}{ }^{-2} \mathrm{~s}^{-1}$ (PAR) y $\mathrm{pH}$ ajustado a 6.1 (Fig. 1). En los experimentos se trabajo únicamente con las raíces nuevas que las plantas recolectadas generaron.

La incorporación de nutrientes en las raíces de $S$. perennis fue estimada mediante incubaciones con medios base con diferentes concentraciones de sustrato conocidas $\left(\mathrm{NH}_{4}^{+}, \mathrm{NO}_{3}^{-}, \mathrm{PO}_{4}^{3-}\right)$ observándose la variación del sustrato en el medio de experimentación (método de desaparición del sustrato del medio).

Las tasas de incorporación de nutrientes se cuantificaron en raíces de $S$. perennis $(n=3)$ preincubadas en $100 \mathrm{ml}$ del medio base sin el nutriente a medir, durante un periodo de 3-7 días dependiendo del nutriente (3-4 días en el caso del $\mathrm{NO}_{3}^{-}$y $\mathrm{NH}_{4}^{+}, 6-7$ días en el caso del $\mathrm{PO}_{4}^{3-}$ ).

El medio base tuvo la siguiente composición: $500 \mathrm{mM} \mathrm{NaCl}, 10 \mathrm{mM} \mathrm{KCl}, 12 \mathrm{mM} \mathrm{CaCl}_{2}$, $55 \mathrm{mM} \mathrm{MgCl} 2,2 \mathrm{mM} \mathrm{NaHCO} 3$ fue tamponado a pH 6.1 con una solución de $\mathrm{NaOH} 200 \mu \mathrm{M}$, y agitado e aireado continuamente.
A este medio se le añadían diferentes concentraciones del nutriente a medir $\left(\mathrm{NO}_{3}^{-}, \mathrm{PO}_{4}^{3-}, \mathrm{NH}_{4}^{+}\right)$; y se obtenían registros de la evolución temporal de la concentración de nutrientes en el medio de incubación.

Las incubaciones se realizaron sobre periodos de tiempo de 24 horas, utilizándose matraces de $250 \mathrm{ml}$ en agitación continua a $25^{\circ} \mathrm{C}$ que contenían $100 \mathrm{ml}$ de medio de ensayo con una biomasa radicular de aproximadamente $0.3 \mathrm{~g}$ de peso fresco, tomándose muestras de medio a los $0,10,30$ minutos y a 1, 2, 4, 5 y 24 horas de incubación.

Las muestras fueron analizadas posteriormente para obtener la concentración de fosfato, nitrato o amonio, mediante los métodos del verde malaquita de Fernández et al., 1985, método industrial 818-871 (basado en los de Shinn, 1941 y Wood et al., 1967), y método 786-861 (basado en el de Slawyk y MacIsaac, 1972) respectivamente, todos en su versión automatizada para un autoanalizador Technicon AAII.

Los ajustes de las cinéticas de incorporación de nutrientes se llevaron a cabo mediante el programa informático Kaleidagraph 4. Se realizaron ANOVAS de una vía para comprobar si las diferencias entre las velocidades de incorporación de los nutrientes, las constantes de semisaturación y los puntos de compensación del sustrato respectivamente obtenidas eran estadísticamente significativas dependiendo del nutriente suministrado. Toda la estadística se realizó mediante el programa informático Statgraphics 3.1.

\section{RESULTADOS}

Los experimentos en cultivos hidropónicos, reflejaron la incorporación de fosfato, amonio y nitrato, a partir de concentraciones iniciales de los sustratos diferentes.

La variación de la concentración de los nutrientes en los distintos cultivos durante el periodo de experimentación (24 horas) no sigue un patrón lineal, de manera que las tasas de incorporación resultaron máximas al inicio de las incubaciones y disminuyeron a medida que transcurría el tiempo.

Tanto para el $\mathrm{PO}_{4}^{3-}, \mathrm{NO}_{3}^{-}, \mathrm{NH}_{4}^{+}$, del ajuste lineal de la concentración de sustrato en el tiempo medida durante las 5 primeras horas de incubación, se 
(i)
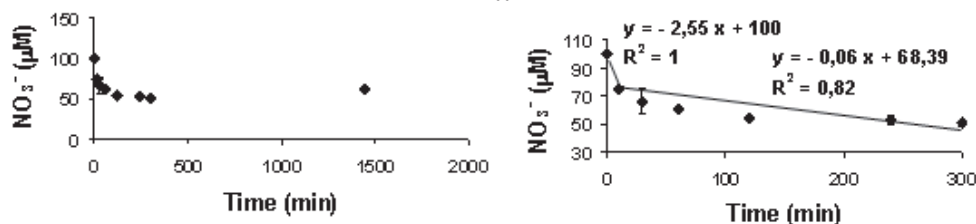

(ii)
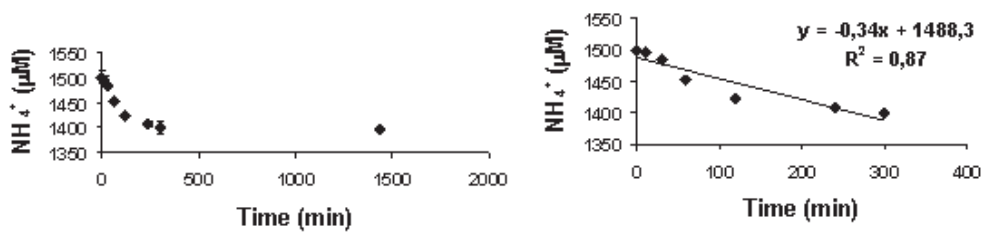

(iii)
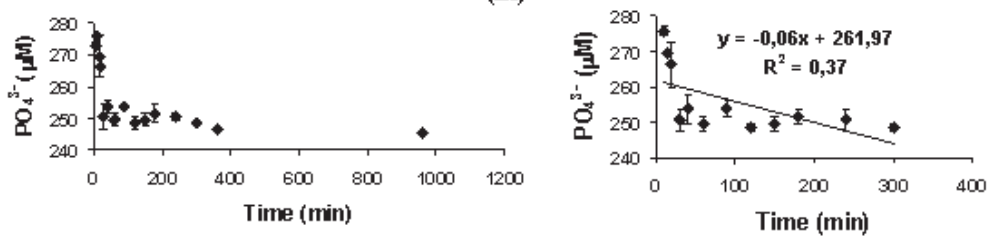

Figura 2. Desaparición del sustrato del medio de cultivo y ajuste lineal para la estimación de tasas de incorporación máximas de cada nutriente: (i) $\mathrm{NO}_{3}^{-}$(ii) $\mathrm{NH}_{4}^{+}$(iii) $\mathrm{PO}_{4}^{3-}$. Substrate disappearance in the culture medium and linear adjustment for the estimation of maximum uptake rates of every nutrient: (i) $\mathrm{NO}_{3}^{-}$(ii) $\mathrm{NH}_{4}^{+}$(iii) $\mathrm{PO}_{4}^{3-}$.

obtienen pendientes que estiman la velocidad de incorporación del nutriente a una concentración determinada, antes de que entren en juego mecanismos de inhibición. Estas tasas de incorporación fueron calculadas y relativizadas al peso de raíz utilizada y proporcionan las tasas de incorporación de cada nutriente ( $\mu$ moles $g^{-1}$ p.s.raíz $\mathrm{h}^{-1}$ ).

Los valores de las tasas de incorporación máxima $\left(V_{\max }\right)$, las constantes de semisaturación ó afinidad $\left(K_{m}\right)$ y los puntos de compensación para cada sustrato $\left(C_{\min }\right)$ fueron estimadas por ajuste de los datos mediante el modelo de EdwardsWalkers a una cinética de Michaelis-Menten,

$$
V=V_{\max }\left(C-C_{\min }\right) / K_{m}+C
$$

donde $V$ es la tasa de incorporación para una concentración de sustrato $C, V_{\max }$ es la tasa de incorporación máxima a concentraciones saturantes del sustrato, $C_{\min }$ es el punto de compensación para el sustrato ó concentración de sustrato a la cual no hay todavía incorporación ( $V=0)$ y $K_{m}$ es la constante de semisaturación, se define como la concentración de sustrato donde la $V=(1 / 2) V_{\max }$.

Los resultados de incorporación estimados por medida de la disminución de las concentraciones de fosfato, amonio y nitrato en el medio se presentan en la figura 2, indicando la incorporación de éstos a través de las raíces de $S$. perennis.

Tabla 1. Efecto de la fuente de nutriente: $\mathrm{NO}_{3}^{-}, \mathrm{NH}_{4}^{+}, \mathrm{PO}_{4}^{3-}$; sobre los parámetros (media $\pm \mathrm{sd} ; n=3$ ) de las cinéticas de incorporación de Sarcocornia perennis. Effect of the nutrient source: $\mathrm{NO}_{3}^{-}, \mathrm{NH}_{4}^{+}, \mathrm{PO}_{4}^{3-}$; on the parameters (means $\pm s d$; $\mathrm{n}=3$ ) of Sarcocornia perennis uptake kinetics.

\begin{tabular}{lccc}
\hline & \multicolumn{3}{c}{ Fuente del Nutriente } \\
\cline { 2 - 4 } & $\mathrm{NO}_{3}^{-}$ & $\mathrm{NH}_{4}^{+}$ & $\mathrm{PO}_{4}^{3-}$ \\
\hline$V_{\max }\left(\mu \mathrm{mol} \mathrm{g} \mathrm{P.S.} .^{-1}{\left.\text { raíz } \mathrm{min}^{-1}\right)}\right.$ & $0.62( \pm 0.03)$ & $1.57( \pm 0.08)$ & $0.055( \pm 0.005)$ \\
$K_{m}(\mu \mathrm{M})$ & $63.94( \pm 9.95)$ & $205.31( \pm 50.27)$ & $28.4( \pm 9.36)$ \\
$C_{\min }(\mu \mathrm{M})$ & $4.02( \pm 1.17)$ & $65.221( \pm 13.92)$ & $1.8( \pm 0.68)$ \\
\hline
\end{tabular}



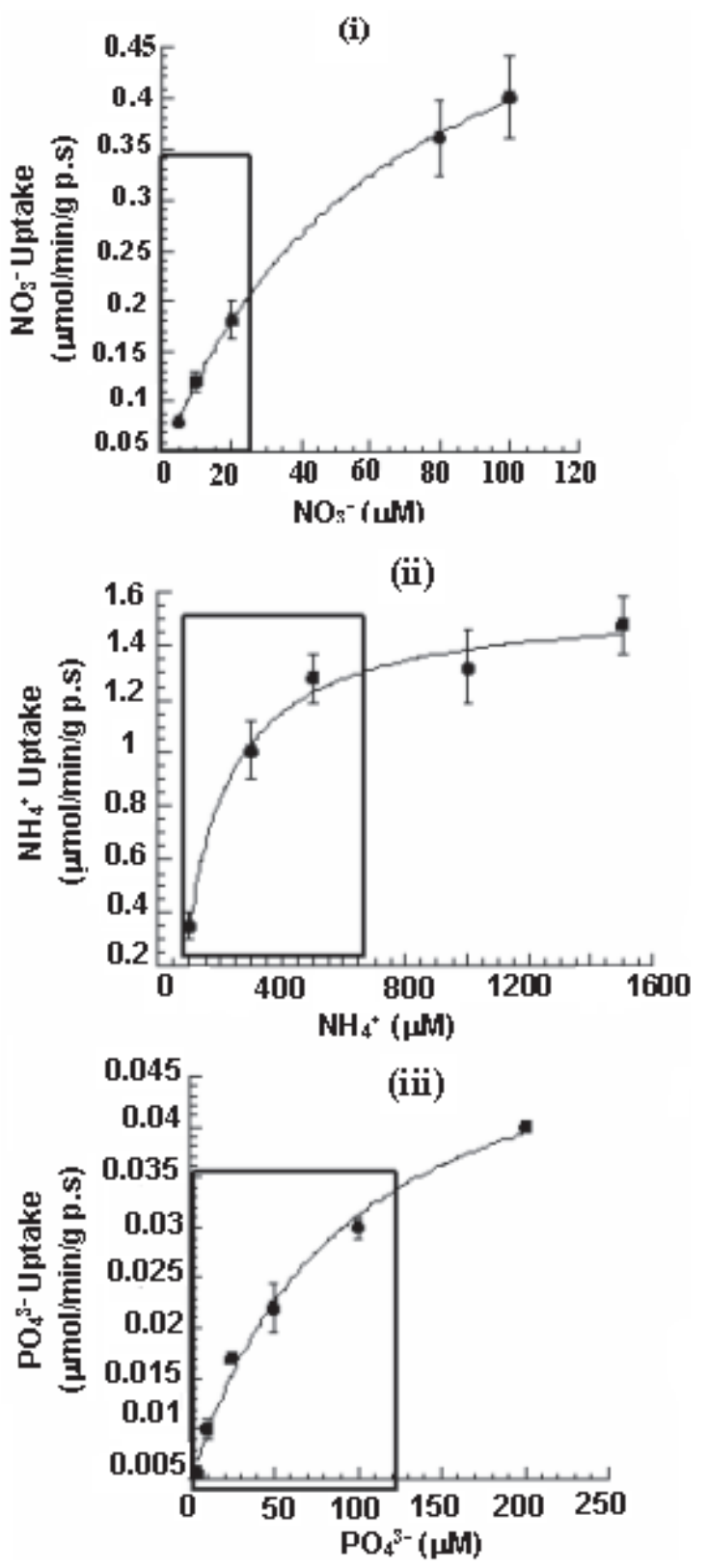

Figura 3. Cinéticas de incorporación de nutrientes por Sarcocornia perennis ajustadas mediante el modelo de ajuste de Edwars-Walker a cinética de Michaelis-Menten: (i) $\mathrm{NO}_{3}^{-}$ (ii) $\mathrm{NH}_{4}^{+}$(iii) $\mathrm{PO}_{4}^{3-}$. Tasas de incorporación expresadas en

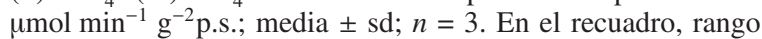
de variabilidad natural registrado para cada nutriente en el estuario del río Palmones. Nutrient uptake kinetics for Sarcocornia perennis fitted by means of the model of Edwars's-Walker adjustment to Michaelis-Menten kinetic: (i) $\mathrm{NO}_{3}^{-}$(ii) $\mathrm{NH}_{4}^{+}$(iii)

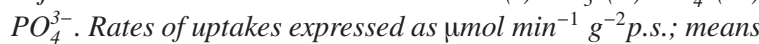
$\pm s d ; \mathrm{n}=3$. In the repicture, range of natural variability registered for every nutrient in the Palmones River Estuary.
Las cinéticas de incorporación para cada nutriente se muestran en la figura 3. Este tipo de cinéticas indica la existencia de un transporte activo de $\mathrm{PO}_{4}^{3-}, \mathrm{NO}_{3}^{-}, \mathrm{NH}_{4}^{+}$. Los parámetros de incorporación se muestran en la tabla 1.

Los resultados de los ANOVAS mostraron diferencias estadísticamente significativas entre las $V_{\max }$ y $K_{m}(P=0.004$ y $P=<0.001$ respectivamente), dependiendo de la fuente de nutriente suministrada, pero no se encontraron diferencias estadísticamente significativas entre las concentraciones de sustrato mínima para que se comience la incorporación $(P=0.050)$.

\section{DISCUSIÓN}

En este estudio se determinan las cinéticas de incorporación de tres fuentes de nutrientes para $S$. perennis, y se presentan resultados de incorporación de nutrientes tanto de nitrógeno $\left(\mathrm{NH}_{4}^{+}\right.$y $\mathrm{NO}_{3}^{-}$) como de fósforo $\left(\mathrm{PO}_{4}^{3}\right)$.

La importancia de este estudio radica en que por primera vez se determinan las tasas de incorporación de nutrientes para $S$. perennis, ya que en la bibliografía no se encuentran datos de incorporación de nutrientes por este tipo de plantas, y constituye una interesante fuente de datos para los estudios de dinámica de los nutrientes en el estuario del río Palmones.

Además, la novedad que presentamos, no sólo es estimar la incorporación de nutrientes por esta planta de marisma, sino estimar la incorporación de los nutrientes en concentraciones reales que encontramos en la marisma, a diferencia de otros experimentos realizados sobre incorporación de nutrientes que sobreestimaban las concentraciones en el medio, y por tanto pueden sobreestimar las tasas de incorporación que se dan en realidad.

Las cinéticas de incorporación de nitrógeno $\left(\mathrm{NH}_{4}^{+}\right.$y $\left.\mathrm{NO}_{3}^{-}\right)$en plantas acuáticas están bien estudiadas (Spartina alterniflora y Spartina patens (Morris, 1980, 1984); Salvinia natans (Jampeetong y Brix, 2009); Eichhornia crassipes (Imaoka y Teramishi, 1988); Vallisneria spiralis L. (Gentner, 1977); Vallisneria americana (Wigand y Stevenson, 1987)). En vegetación de marisma, se han realizado estudios de incorporación en dos especies 
del género Spartina (Spartina patens y Spartina alterniflora) (Morris 1980; Morris 1984).

Respecto del fósforo, se encuentran datos de estudios realizados sobre incorporación de nutrientes de fósforo en macrófitos (Elodea canadensis (Pelton et al., 1998), fanerógamas marinas (Zostera marina (Mc Roy y Barsdate, 1970; McRoy et al., 1972; Murray et al., 1992; Brix y Lyngby, 1985 ); Zostera noltii (Pérez-Llorens y Niell, 1995); Thalassia testidinum (Gras et al., 2003)), plantas acuáticas (Myriophuyllum spicatum y Hydrilla verticillata (Bole \& Allan, 1978); Azolla caroliniana willd y Salvinia rotundifolia willd (Debusk \& Redy, 1987)), macroalgas (Enteromorpha intestinales (Cohen \& Fong, 2004)), aunque nada se encuentra en la bibliografía sobre incorporación de nutrientes de fósforo en vegetación de marisma.

En los experimentos de incubación con el sustrato, S. perennis respondió de diferente forma cuando se le suministraron diferentes formas de $\mathrm{N}$ y P inorgánico $\left(\mathrm{NO}_{3}^{-}, \mathrm{NH}_{4}^{+}, \mathrm{PO}_{4}^{3-}\right)$.

Los datos sugieren que $S$. perennis presenta una mayor afinidad por el fosfato que por los nutrientes de nitrógeno. El fosfato se encuentra en concentraciones en la marisma muy por encima de la concentración mínima necesaria para que comience la incorporación $\left(C_{\min }\right)$ (Tabla 1$)$, por lo que este nutriente no constituiría un factor limitante.

En condiciones de saturación, $S$. perennis asimila fosfato con un flujo máximo de $3.3 \mu$ moles $P$ $\mathrm{g}^{-1}$ p.s. raíz $\mathrm{h}^{-1}$, si comparamos este flujo con los encontrados en otros estudios, obviando las diferencias entre tipos de vegetación, observamos que $S$. perennis se presenta como una especie con gran afinidad por la incorporación de fósforo $\left(\mathrm{PO}_{4}^{3-}\right)$ en la marisma, en comparación con otros datos de incorporación de fósforo obtenidos cuyos valores son mucho menores por ejemplo, Brix y Lyngby (1985) hallaron valores de incorporación de fosfato por la fanerógama marina Zostera marina, de $0.012 \pm 0.007 \mu$ g fosfato $\mathrm{g}_{P . S}^{-1}$; Debusk \& Reddy (1987) encontraron en Azolla caroliana willd y Salvinia rotundifolia, tasas de incorporación de $21.4 \mathrm{mg} \mathrm{m}^{-2}$ día $^{-1}$ y $26.5 \mathrm{mg} \mathrm{m}^{-2}$ día $^{-1}$ respectivamente $(2.56 \mathrm{~g}$ $\mathrm{m}^{-2}$ día $^{-1}$ para Sarcocornia); Imaoka y Teranishi (1988) estimaron tasas de $0.78 \mathrm{mg} \mathrm{P} \mathrm{g}^{-1}$ p.s.día ${ }^{-1}$ en la planta acuática Eichhornia crassipes,; Pérez-Llorens (1991) encontraron tasas de incorporación para Zostera noltii de 2.60 umoles $\mathrm{g}^{-1}$ p.s.raíz $\mathrm{h}^{-1}$; etc. Respecto a la constante de semisaturación, Pérez-Llorens (1991) encuentra valores de $K_{m}$ de $2.67 \mu \mathrm{M}$ para Zostera noltii, lo cual indica una afinidad de Zostera por el fosfato mayor que Sarcocornia (Tabla 1), aunque hoy en día ya no se encuentran praderas de esta fanerógama en el estuario (Clavero et al., 1999).

Por otro lado, los resultados muestran que $S$. perennis, muestra la capacidad de asimilar nitrógeno por la raíces en forma de $\mathrm{NO}_{3}^{-}$y $\mathrm{NH}_{4}^{+}$, aunque la forma de nitrógeno que mayor afinidad presenta para ser asimilada es el amonio. El nitrato en el agua intersticial del sedimento de la marisma se encuentra en concentraciones bajas (0.04-17.45 $\mu \mathrm{M})$, llegando a ser en ocasiones menor que la concentración mínima necesaria para que comience su incorporación, además la concentración idónea $\left(K_{m}\right)$ para la incorporación este nutriente, se encuentra muy por encima de las concentraciones que se encuentran en el agua intersticial, por lo que el nitrato puede presentarse como factor limitante para $S$. perennis en la marisma.

El nitrato como fuente de nitrógeno puede ser sustituido por el amonio, éste se encuentra en el sedimento en concentraciones muy superiores (134-1505 $\mu \mathrm{M})$ a $C_{\min }$, y a $K_{m}$ (Tabla 1 ), lo que lo convierte en la forma de nitrógeno más asequible, supliendo así la limitación de nitrógeno por el nitrato. Las velocidades de incorporación del nitrato y el amonio en la marisma también han sido estimadas, encontrándose tasas de incorporación de $0.62 \mu$ moles $\mathrm{g}^{-1}$ p.s.raíz min $^{-1}$ y $1.57 \mu$ moles $g^{-1}$ p.s.raíz min $^{-1}$ respectivamente (ó lo que es equivalente: $37.2 \mu$ moles $\mathrm{g}^{-1}$ p.s.raíz $\mathrm{h}^{-1}$ para el nitrato y $94.2 \mu$ moles $\mathrm{g}^{-1}$ p.s.raíz $\mathrm{h}^{-1}$ para el amonio). Si se compara la tasa de incorporación del amonio encontrada para $S$. perennis con la tasas obtenidas por Morris (1984) para otras plantas de marisma como son Spartina alterniflora ó Spartina patens $(24 \pm$ 3.8 umoles $g^{-1}$ p.s.raíz $\mathrm{h}^{-1}$ y $27.67 \pm 1.3$ umoles $\mathrm{g}^{-1}$ p.s.raíz $\mathrm{h}^{-1}$ respectivamente), la tasa de incorporación de amonio que presenta Sarcocornia es mucho mayor que la que presentan ambas especies de Spartina lo que refleja la gran capacidad 
de la quenopodiácea para asimilar el nitrógeno en forma de amonio que se encuentran en los barros reducidos de la marisma. También hay que tener en cuenta la concentración suministrada a las especies; mientras que Morris $(1982,1984)$ utilizaban concentraciones de sustrato en sus experimentos entorno a los $500 \mu \mathrm{M}$ de $\mathrm{NO}_{3}^{-}$ó $\mathrm{NH}_{4}^{+}$ respectivamente, para obtener las tasas de incorporación máximas; aquí se utilizan concentraciones muy superiores (de hasta $1500 \mu \mathrm{M}$ en el caso del amonio y $100 \mu \mathrm{M}$ en el caso del nitrato), quizá sea ésta la causa de las marcadas diferencias en las tasas máximas de incorporación entre ambos tipos de plantas, sin tener en cuenta las características fisiológicas de cada una de ellas, lo que si se puede extraer de este estudio es la mayor eficiencia de Sarcocornia respecto a Spartina para subsistir en un medio eutrófico como es el del estuario del río Palmones, y quizá sea este el motivo por el cual siendo Spartina una planta típica de marisma, no se encuentra en el estuario del río Palmones.

Por otro lado, diversos autores demuestran síntomas de toxicidad por amonio en plantas que aparecen en concentraciones de amonio exteriores por encima de 0.1-0.5 mM (Britto y Kronzucker, 2002; Tylova et al., 2008). En el presente estudio, sin embargo, el suministro de concentraciones de amonio externas de hasta $1.5 \mathrm{mM}$, no afectó a los mecanismos de incorporación de $\mathrm{NH}_{4}^{+}$por $S$. perennis, indicando la posibilidad de la adaptación de esta especie a las altas concentraciones de amonio que se registran en el agua intersticial de las marismas del Palmones.

Además, existen estudios que hablan de cómo factores como la temperatura, la salinidad, la oxigenación del suelo, etc. pueden variar la velocidad máxima de incorporación de los nutrientes; sin embargo, los valores de Km deben permanecer constantes según los resultados obtenidos por Lycklama (1963) y van den Honert y Hooymans (1955). Aunque desde este estudio no se apoya esta la teoría sobre la constancia de $K_{m}$, en nuestro caso, no se puede estimar el efecto de estas variables sobre las tasas de incorporación ya que todos los experimentos se realizaron con condiciones de salinidad, temperatura, y oxigenación constante y controlada.

\section{AGRADECIMIENTOS}

Este trabajo ha sido subvencionado por la Consejería de Innovación Ciencia y Empresa de la Junta de Andalucía, mediante el proyecto P06-RNM-01892.

\section{BIBLIOGRAFÍA}

AVILÉS, A. 2002. El papel del sector fluvial en la biogeoquímica del río Palmones (Los Barrios, Cádiz). Tesis doctoral. Universidad de Málaga. 191 pp.

BOLE J. B. \& J. R. ALLAN. 1978. Uptake of phosphorus from sediment by aquatic plants, Myriophyllum spicatum and Hydrilla veticillata. Water Res., 12: 353-358.

BRITTO, D. T. \& H. J. KRONZUCKER. 2002. $\mathrm{NH}_{4}^{+}$ toxicity in higher plants: a critical review. J. Plant Physiol., 159: 567-584.

BRIX, H. \& J. E. LYNGBY. 1985. Uptake and translocation of phosphorus in eelgrass (Zostera marina). Marine Biology, 90: 111-116.

CAETANO, M., C. VALE, R. CESÁRIO \& N. FONSECA. 2008. Evidence for preferential depths of metal retention in roots of salt marsh plants. Science of the Total Environment, 390: 466-474.

CEDERGREEN, N. \& T. V. MADSEN. 2003a. Nitrate reductase activity in roots and shoots of aquatic macrophytes. Aquat. Bot., 76: 203-212.

CLAVERO, V., J. J. IZQUIERDO, J. A. FERNÁNDEZ \& F. X. NIELL. 2000. Seasonal fluxes of phosphate and ammonium across the sedimentwater interface in a shallow small estuary (Palmones River, southern Spain). Marine Ecology Progress Series, 198: 51-60.

CLAVERO, V., J. J. IZQUIERDO, L. PALOMO, J. A. FERNÁNDEZ \& F. X. NIELL. 1999b. Water management and climate changes increase the phosphorus accumulation in the small shallow estuary of Palmones River (southern Spain). Science of the Total Environment, 228: 193-202.

COHEN, R. A. \& P. FONG. 2004 Nitrogen uptake and assimilation in Enteromorpha intestinalis (L.) Link (Chlorophyta): using $15 \mathrm{~N}$ to determinate preference during simultaneous pulses of nitrate and ammonium. Journal of Experimental Marine Biology and Ecology, 309: 67-77.

DEBUSK, W. F. \& K. R. REDDY. 1987. Growth and nutrient uptake potential of Azolla caroliniana Wi- 
1ld. And Salvinia rotundifolia Willd. As a function of temperature. Environmental and Experimental Botany, 27: 215-221.

DE LAUNE, R. D., C. J. SMITH \& W. H. PATRICK. 1983. Nitrogen losses from a Louisiana Gulf Coast salt marsh. Estuarine, Coastal and Shelf Science, 17: 133-141.

EPSTEIN, E. 1972. Mineral Nutrition of Plants: Principles and Perspectives. Wiley, New York: John Wiley. \&. Sons. 412 pp.

FERNÁNDEZ, J. A., F. X. NIELL \& J. LUCENA, J. 1985. A rapid and sensitive automated determination of phosphate in natural waters. Limnol. Oceanogr. 30: 227-230.

FRIED, M., F. ZSOLDOS, P. B. VOSE \& I. L. SHATOKHIN. 1965. Characterizing the $\mathrm{NO}_{3}^{-}$and $\mathrm{NH}_{4}^{+}$ uptake process of rice roots by use of $15 \mathrm{~N}$ labelled $\mathrm{NH}_{4}$ and $\mathrm{NO}_{3}$. Physiologia Plantarum, 18: 313-320.

GENTNER, S. R. 1977. Uptake and transport of iron and phosphate by Vallisneria spiralis L. Aquatic Botany, 3: 267-272.

GRAS, A. G., M. S. KOCH \& C. J. MADDEN. 2003. Phosphorus uptake kinetics of a dominant tropical seagrass Thalassia testidinum. Aquatic Botany, 76: 299-315.

HAINES, K. C. \& P. A. WHEELER. 1978. Ammonium and nitrate uptake by the marine macrophytes Hypnea musciformis (Rhodophyta) and Macrocystis pyrifera (Phaeophyta). J. Phycol., 14: 319-324.

HAMMOND, I. C., W. H. ALLAWAY \& W. E. LOOMIS. 1955. Effects of oxygen and carbon dioxide levels upon absorption of potassium by plants. Plant Physiol., 30: 155-161.

HELAL, M., K. KOCH \& K. MENGEL. 1975. Effect of salinity and potassium on the uptake of nitrogen and on nitrogen metabolism in young barley roots. Physiolgia Plantarum, 35: 310-313.

HOPKINS, H. T. 1956. Absorption of ionic species of orthophosphate by barley roots: effects of 2,4dinitrophenol and oxygen tension, Plant Physiol., 31: 155-161.

IMAOKA, T. \& S. TERANISHI. 1988. Rates of nutrient uptake and growth of the water Hyacinth (Eichhornia crassipes (Mart.) Solms). Wat. Res., 22: 943-951.

IZQUIERDO, J. J. 2001. Aspectos del ciclo de los nutrientes no conservativos en el estuario del río Palmones. Tesis doctoral. Universidad de Málaga. $233 \mathrm{pp}$.
JAMPEETONG, A. \& H. BRIX. 2009. Nitrogen nutrition of Salvinia natans: Efffects of inorganic nitrogen form on growth, morphology, nitrate reductase activity and uptake kinetics of ammonium and nitrate. Aquatic Botany, 90: 67-73.

LOTZE, H. K. \& W. SCHRAMM. 2000. Ecophysiological traits explain species dominance patterns in macroalgal blooms. J. Phycol., 36: 287-295.

LYCKLAMA, J. C. 1963. The absorption of ammonium and nitrate by perennial rye-grass. Acta Bot. Neerl., 12: 361-423.

MCROY, C. P. \& R. J. BARSDATE. 1970. Phosphate absorption in eelgrass. Limnol. Oceanogr., 15: 6-13.

MCROY, C. P., R. J. BARSDATE \& M. NEBERT. 1972. Phosphorus cycling in an eelgrass (Z. marina L.) ecosystem. Limnol. Oceanogr., 17: 58-67.

MENDELSON, I. A. 1979. Nitrogen metabolism in the height forms of Spartina alterniflora in North Carolina. Ecology, 60: 574-584.

MORRIS, J. T. 1980. The nitrogen uptake kinetics of Spartina alterniflora in culture. Ecology, 61: 11141121.

MORRIS, J. T. 1984. Efects of nitrogen and salinity on ammonium uptake by Spartina alterniflora Loisel. and Spartina Patens (Aiton) Muhl. J. Exp. Mar. Biol. Ecol., 78: 87-98.

MURRAY, L., W. C. DENINSON \& W. M. KEMP. 1992. Nitrogen versus phosphorus limitation for growth of an estuarine population of eelgrass (Zostera marina L.). Aquatic Botany, 44: 83-100.

NIELL, F. X., C. FERNÁNDEZ, F. L. FIGUEROA, F. G. FIGUERIAS, J. M. FUENTES, J. L. PÉREZLLORENS, M. J. GARCÍA, I. HERNÁNDEZ, J. A. FERNÁNDEZ, M. ESPEJO, J. BUELA, M. C. GARCÍA-JIMÉNEZ, V. CLAVERO \& C. JIMÉNEZ. 1996. Spanish Atlantic coasts. In: $M a-$ rine benthic vegetation. W. Schramm \& P. H. Nienhuis (eds.).: 265-281. Springer-Verlag, Berlin.

PALOMO, L. 2004. La influencia de Sarcocornia perennis alpini (Lag.) Castroviejo en el flujo de nutrientes del estuario del río Palmones. Tesis doctoral, Universidad de Málaga. 225 pp.

PELTON, D. K., S. N. LEVINE \& M. BRANER. 1998. Measurements of phosphorus uptake by macrophytes and epiphytes from the LaPlatte River (VT) using 32P in stream microcosms. Freshwat. Biol., 39: 285-299.

PEREZ-LLORENS, J. L. 1991. Estimaciones de biomasa y contenido interno de nutrients, ecofisiología de incorporación de carbono y fosfato en 
Zostera Noltii Hormem. Tesis doctoral, Universidad de Málaga. 168 pp.

PEREZ-LLORENS，J. L. \& F. X. NIELL. 1995. Short term phosphate uptake kinetics in Zostera noltii Hornem: a comparision between excised leaves and sediment rooted plants. Hydrobiologia, 297: 17-27.

RAO, D. N. \& D. S. MIKKELSEN. 1977. Effects of $\mathrm{CO}, \mathrm{CH}$, and $\mathrm{N}$, on growth and nutrition of rice seedlings. Plant Soil, 47: 313-322.

RAYAR, A. J. \& T. HAI. 1977. Effect of ammonium on uptake of phosphorus, potassium, calcium and magnesium by intact soybean plants. Plant Soil, 48: 81-87.

ROSENBERG, G. \& J. RAMUS. 1984. Uptake of inorganic nitrogen and seaweed surface area:volume ratios. Aquat. Bot., 19: 65-72.

SHINN, J. A. 1941. Ind. Eng. Chem. (Annual edition), 13:33. In: A practical approach handbook of seawater analysis. J. D. H. Strickland \& T. R. Parson (eds.). Ottawa. Fish Res. Bd. Can. 310 pp.

SIMAS, T. C. \& J. G. FERREIRA. 2007. Nutrient enrichment and the role of salt marshes in the Tagus estuary (Portugal). Estuarine, Coastal and Shelf Science, 75: 393-407.

SLAWIK, G. \& J. J. MACISAAC. 1972. Comparison of two automated ammonium methods in a region of coastal upwelling. Deep-Sea Research, 19: 521524.
SMITH, W. F. \& W. A. JACKSON. 1998. Nitrogen enhancement of phosphate transport in root of Zea mays L. Plant Physiol., 84: 1319-1324.

TOUCHETTE, B. W. \& J. M. BURKHOLDER. 2000 Review of nitrogen and phosphorus metabolism in seagrasses. Journal of Experimental Marine Biology and Ecology, 250: 133-167.

TYLOVA, E., L. STEINBACHOVA, O. VOTRUBOVA, B. LORENZEN \& H. BRIX. 2008. Different sensitivity of Phragmites australis and Glyceria maxima to high availability of ammonium-N . Aquat. Bot., 88: 93-98.

VAN DEN HONERT, T. H. \& J. J. H. HOOYMANS. 1955. On the absorption of nitrate by maize in water culture. Acta Bot. Neerl., 4: 376-384.

VLAMIS, J. \& A. R. DAVIS. 1944. Effects of oxygen tension on certain physiological responses of rice, barley and tomato. Plant Physiol., 19: 33-51.

WAITE, T. \& R. MITCHELL. 1972. The effect of nutrient fertilization on the benthic alga Ulva lactuca. Bot. Mar. 25: 151-156.

WIGAND, C. \& J. C. STEVENSON. 1997 Facilitation of phosphate assimilation by aquatic mycorrhizae of Vallisneria americana Michx. Hydrobiologia, 342/343: 35-41.

WOOD, E. D., F. A. J. AMSTRONG \& F. RICHARDS. 1967. Determination of nitrate in seawater by cadmium-copper reduction to nitrite. Journal of Marine Biology, 47: 23-31. 